\title{
Duodenal Perforation, CTCAE
}

National Cancer Institute

\section{Source}

National Cancer Institute. Duodenal Perforation, CT CAE. NCI Thesaurus. Code C143417.

A disorder characterized by a rupture in the duodenal wall. 